\title{
EL RUMBO DE LA POBLACIÓN DESPLAZADA EN BOGOTÁ-COLOMBIA. Estudio de caso en la Localidad de Kennedy, 1980-2016.
}

\author{
The Course of the Population Displaced in Bogota-Colombia. Case study in the \\ locarity of Kennedy, 1980-2016.
}

\author{
Hugo Andrés Granados Castillo \\ Pedro Oswaldo Loaiza Parra \\ Director: Dr. Oscar Luis Pyszczek \\ Programa de Ingeniería Geográfica y Ambiental. Facultad de Ingeniería. Universidad \\ de Ciencias Aplicadas y Ambientales (UDCA)-Colombia.
}

http://dx.doi.org/10.30972/geo.14282744

\section{RESUMEN}

El objeto de este estudio es identificar y analizar los diferentes flujos de emigración de la población afectada por el desplazamiento forzado hacía la ciudad de Bogotá. Inicialmente, se desarrolló un estudio de caso de tipo exploratorio que consistió en visitar diferentes entidades públicas y privadas, con el fin de recolectar información cuantitativa sobre familias desplazadas y que decidieron asentarse en las localidades de Bogotá. Al ser un estudio socio témporo-espacial fue pertinente trabajar desde 1980 hasta el 2016. Posteriormente se realizó un procesamiento de información espacial donde se evidencia el aumento de la población desplazada en las diferentes localidades de Bogotá, como soporte se realizaron gráficos que permitieron generar un contraste de información. Finalmente se analizaron los resultados obtenidos y al cruzar variables sociales se concluye, que la solución por parte de las entidades no debe ser meramente asistencial, sino que debe tener un enfoque en los derechos humanos.

\section{PALABRAS CLAVES}

Conflicto armado; Localidad; Socio témporo-espacial; Población desplazada; Emigración.

\section{ABSTRACT}

The object of this investigation to consist in identify and analize the diferent flow of emigration to the population affected for the forced displacement toward Bogota city. Firtsly, we worked in a exploratory case study, it's consisted the visit to the diferent publics and privates organizations to colect cuantitative information about the families that suffered forced displacement and decided settle in the diferents Bogota's localities. For this sociotemporary and spacial study was pertinent work from 1980 to 2016, Subsequently, a spatial information processing was carried out where the increase of the displaced population in the different localities of Bogotá is evidenced, as support was made graphics that allowed to generate a contrast of information. Finally the answers to analize to get social variables to allow that the solution of this part to the differents entities dont must to be just asistential, it should be to have a focus in the human rights.

\section{KEY WORDS}

Armed conflicto; Locality; Socio temporary-spacial; Population ousted; Emigration.

Publicado en formato digital: Hugo A. Granados Castillo, Pedro O. Loaiza Parra (Director: Dr. Oscar Luis Pyszczek). EL RUMBO DE LA POBLACIÓN DESPLAZADA EN BOGOTÁ-COLOMBIA. Estudio de caso en la Localidad de Kennedy, 1980-2016. Revista Geográfica Digital. IGUNNE. Facultad de Humanidades. UNNE. Año 14. N² 28. Julio - Diciembre 2017. ISSN 1668-5180 Resistencia, Chaco.

En: http://hum.unne.edu.ar/revistas/geoweb/default.htm - http://dx.doi.org/10.30972/geo.14282744 
Revista Geográfica Digital. IGUNNE. Facultad de Humanidades. UNNE. Año 14. № 28.

Julio - Diciembre 2017. ISSN 1668-5180 Resistencia, Chaco

\section{INTRODUCCIÓN}

Colombia ha sido un país víctima del desplazamiento forzado a causa del conflicto armado, según la Comisión Histórica del Conflicto y sus Víctimas, integrada por doce expertos. No obstante, en Colombia para dar fin al conflicto y surgimiento de los grupos subversivos como el frente nacional que tenían gran dominio sobre el poder político y social, se dio inicio a generar consenso de paz entre el gobierno y estos grupos revolucionarios. Una de las posturas afirma que el conflicto surge al finalizar el frente nacional entre los años (1958 y 1960) y se acrecienta con el nacimiento del narcotráfico en el año de 1980, a partir de estos años campesinos e indígenas han tenido que abandonar sus tierras, por amenazas, muerte de sus familiares, sometimientos, temor y por tener que huir a diferentes lugares en busca de una nueva vida con oportunidades. De acuerdo con el Alto Comisionado de las Naciones Unidas para los Refugiados (ACNUR), Colombia es uno de los países con mayor número de desplazados en el mundo. En el 2007, había cerca de 4 millones de afectados, siendo así uno de los problemas que ha presentado gran crecimiento en el país. Águilas negras, Rastrojos, Fuerzas Armadas Revolucionarías de Colombia (FARC), Ejército de Liberación Nacional (ELN), Fuerza Pública, son algunos de los responsables de este flagelo. Según el reporte de la Consultoría para los Derechos Humanos y el Desplazamiento (CODHES) en el mismo año 2015 se desplazaron 44.791 personas, de las cuáles Bogotá es la ciudad con mayor recepción con 30.596 personas. Según la Organización de Naciones Unidas (ONU) en el estudio realizado en el 2015 la república de Colombia figura como segunda en la lista de refugiados y desplazados obligados a dejar sus hogares por las guerras o por ser víctimas de persecución con un total de 5,3 millones de casos. Por lo cual, es evidente que la problemática aumentaba conforme pasaban los años.

"Bogotá, como capital del país, también padece el recrudecimiento de un conflicto armado con asiento en los barrios periféricos de la urbe, pero con poder sobre economías lícitas e ilícitas que controlan el comercio, la delincuencia común, la prostitución y el microtráfico. El desplazamiento forzado interno en Bogotá es un fenómeno constante, silencioso y con un alarmante subregistro que expone las graves deficiencias en cuanto a protección de la población desplazada en todo el país." (CODHES, 2014)

El suceso de desplazamiento forzado según la Ley 387/1997 es concebida como persona desplazada en Colombia: La que se ha visto forzada a migrar dentro del territorio nacional abandonando su localidad de residencia o actividades económicas habituales, porque su vida, su integridad física, su seguridad o libertades personales han sido vulneradas o se encuentran directamente amenazadas.

En el último informe de la Comisión Accidental del Desplazamiento, las principales localidades de residencia de esta población son: Ciudad Bolívar $(23 \%)$, Kennedy $(14 \%)$, Bosa (13\%), Usme (8\%), San Cristóbal (6\%), Engativá (5,85\%), Rafael Uribe $(5,45 \%)$, Suba $(4,53 \%)$, Tunjuelito $(3,34 \%)$ y Santafé $(2,83 \%)$. Sin embargo; el Registro Único de Victimas (RUV) Afirma que es la localidad de Kennedy la que ha encabezado la lista de afluencia de población desplazada por conflicto armado en los últimos años.

El propósito del siguiente estudio socio témporo-espacial consistió en identificar las áreas de Bogotá que mayor receptividad de población desplazada a causa del conflicto armado han presentado desde el año 1980 hasta el 2016, por medio de una recolección de datos provenientes de diferentes entidades tanto públicas como privadas, el procesamiento estadístico y espacial, su posterior representación cartográfica y análisis de los datos obtenidos. De esta manera se logró hacer una identificación ilustrativa de las localidades que presentan mayor incidencia y receptividad a lo largo de las últimas décadas, lo cual nos

Publicado en formato digital: Hugo A. Granados Castillo, Pedro O. Loaiza Parra (Director: Dr. Oscar Luis Pyszczek). EL RUMBO DE LA POBLACIÓN DESPLAZADA EN BOGOTÁ-COLOMBIA. Estudio de caso en la Localidad de Kennedy, 1980-2016. Revista Geográfica Digital. IGUNNE. Facultad de Humanidades. UNNE. Año 14. Nº 28. Julio - Diciembre 2017. ISSN 1668-5180 Resistencia, Chaco.

En: http://hum.unne.edu.ar/revistas/geoweb/default.htm 
permitió realizar un contraste con información social, para determinar el grado de vulnerabilidad de esta variable. Además, se presentan dos estudios de caso que responden a las localidades con mayor y menor receptividad de la población caracterizada anteriormente.

\section{METODOLOGÍA}

\begin{tabular}{|c|c|c|}
\hline OBJETIVO & ACTIVIDADES & TÉCNICA \\
\hline $\begin{array}{l}\text { Identificar áreas de la } \\
\text { ciudad de Bogotá con } \\
\text { mayor número de } \\
\text { población asentada a } \\
\text { causa del } \\
\text { desplazamiento } \\
\text { forzado (conflicto } \\
\text { armado). }\end{array}$ & 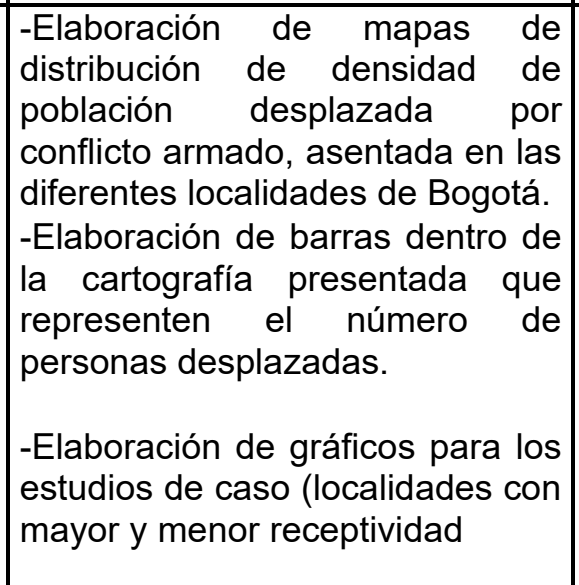 & $\begin{array}{l}\text { 1.Tratamiento estadístico de } \\
\text { información. } \\
\text { 2.Normalización de datos. } \\
\begin{array}{l}\text { 3.Procesamiento } \\
\text { información espacial. } \\
\text { 4.Técnicas de representación } \\
\text { gráfico-cartográficas. }\end{array}\end{array}$ \\
\hline $\begin{array}{l}\text { Interpretación de los } \\
\text { resultados obtenidos a } \\
\text { partir de las técnicas } \\
\text { gráfico-cartográficas. }\end{array}$ & $\begin{array}{l}\text {-Análisis de los gráficos para los } \\
\text { estudios de caso (localidades con } \\
\text { mayor y menor receptividad). }\end{array}$ & $\begin{array}{l}\text { 1.Análisis de variables } \\
\text { cualitativas. } \\
\text { 2.Interpretación de información } \\
\text { obtenida en los gráficos y la } \\
\text { cartografía. } \\
\text { 3. Análisis de los estudios de } \\
\text { caso (localidades con mayor y } \\
\text { menor receptividad). }\end{array}$ \\
\hline $\begin{array}{l}\text { Contraste de variables } \\
\text { espaciales y sociales. } \\
\text { En una temporalidad } \\
\text { de }(1980-2016) \\
\text { representado por } \\
\text { décadas. } \\
\end{array}$ & $\begin{array}{l}\text {-Análisis del cruce de variables } \\
\text { sociales y espaciales. } \\
\text {-Identificaciones de variables } \\
\text { directas (espaciales y sociales). }\end{array}$ & $\begin{array}{l}\text { 1. Interpretación de información } \\
\text { de tipo cuantitativo y cualitativo. }\end{array}$ \\
\hline
\end{tabular}

Tabla 1. Metodología implementada (Elaboración propia)

Publicado en formato digital: Hugo A. Granados Castillo, Pedro O. Loaiza Parra (Director: Dr. Oscar Luis Pyszczek). EL RUMBO DE LA POBLACIÓN DESPLAZADA EN BOGOTÁ-COLOMBIA. Estudio de caso en la Localidad de Kennedy, 1980-2016. Revista Geográfica Digital. IGUNNE. Facultad de Humanidades. UNNE. Año 14. Nº 28. Julio - Diciembre 2017. ISSN 1668-5180 Resistencia, Chaco.

En: http://hum.unne.edu.ar/revistas/geoweb/default.htm 
Revista Geográfica Digital. IGUNNE. Facultad de Humanidades. UNNE. Año 14. № 28.

Julio - Diciembre 2017. ISSN 1668-5180 Resistencia, Chaco

\section{RESULTADOS Y ANÁLISIS}

Inicialmente, para entrar en contexto con la ubicación geográfica, en el siguiente mapa se observa la distribución por localidades de la ciudad de Bogotá.

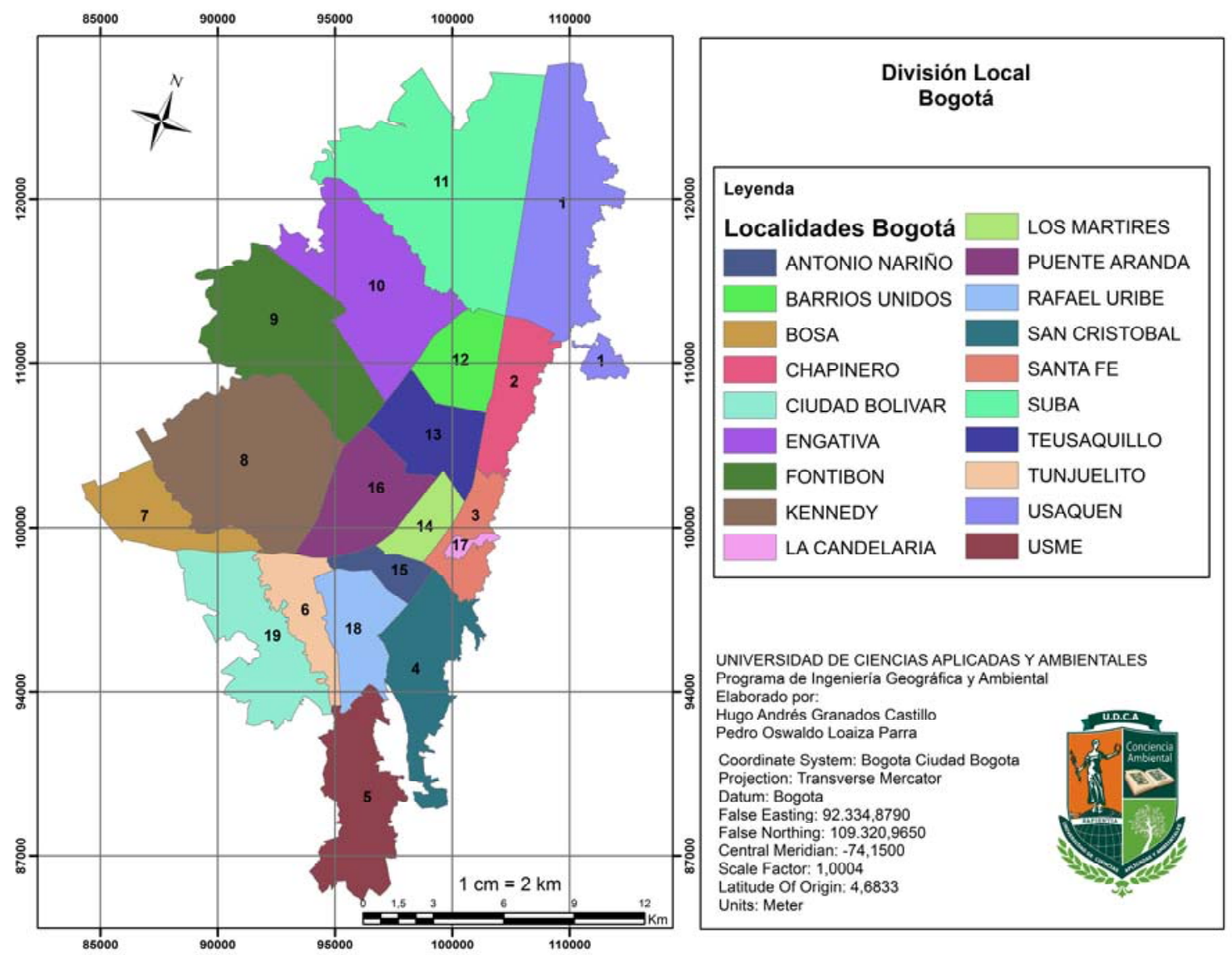

Mapa 1. Distribución de localidades en Bogotá (Elaboración propia, Shapefile obtenido del IDECA Obtenido de https://www.ideca.gov.co/es/servicios/mapa-de-referencia/tabla-mapareferencia)

Publicado en formato digital: Hugo A. Granados Castillo, Pedro O. Loaiza Parra (Director: Dr. Oscar Luis Pyszczek). EL RUMBO DE LA POBLACIÓN DESPLAZADA EN BOGOTÁ-COLOMBIA. Estudio de caso en la Localidad de Kennedy, 1980-2016. Revista Geográfica Digital. IGUNNE. Facultad de Humanidades. UNNE. Año 14. $N^{\circ} 28$. Julio - Diciembre 2017. ISSN 1668-5180 Resistencia, Chaco.

En: http://hum.unne.edu.ar/revistas/geoweb/default.htm 
Revista Geográfica Digital. IGUNNE. Facultad de Humanidades. UNNE. Año 14. № 28.

Julio - Diciembre 2017. ISSN 1668-5180 Resistencia, Chaco

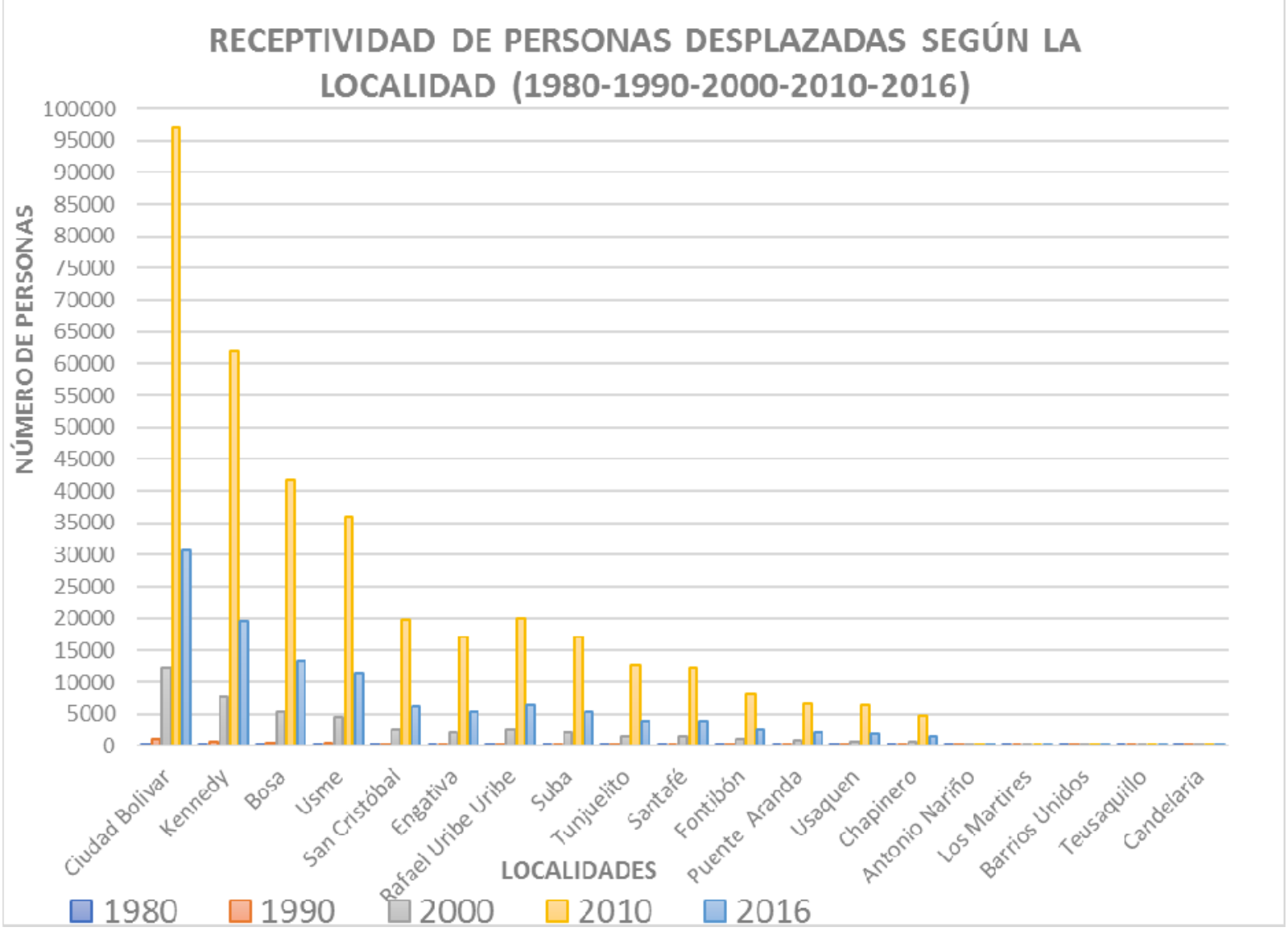

Gráfico 1. Receptividad de Personas Desplazadas según la Localidad 1980-1990-20002010-2016 (elaboración propia con base en los datos del Registro Único de Víctimas (RUV) Obtenido de https://rni.unidadvictimas.gov.co/?q=v-reportes)

En la gráfica anterior es posible observar la relación de las diferentes localidades de Bogotá, las que mayor receptividad han presentado desde 1980 hasta la actualidad están ubicadas desde el sur de la capital hasta el occidente. Una de las principales razones es la baja organización y planeación por parte de las Alcaldías locales, además, si tenemos en cuenta que los mayores índices de pobreza y marginalidad se desarrollan en estas áreas según el último informe del Departamento Administrativo de Estadísticas Nacionales (DANE), la alta receptividad de población desplazada representaría un factor incidente. No obstante, esta situación ha obligado a que las Alcaldías locales integren dentro de sus planes de desarrollo y acción alternativas que respondan a esta problemática, las cuales además de asociarse a los efectos ya mencionados, ocasionan un fenómeno urbano y consigo todos los problemas que genera (aumento de las basuras, agotamiento de los recursos, ruptura de barreras periféricas, contaminación ambiental, entre otros).

Por otro lado, la ubicación geográfica de las localidades juega un papel importante en la receptividad y aumento de la densidad poblacional, las localidades del sur de la capital presentan barreras naturales, que en su mayor medida están siendo alteradas y vulneradas, con el fin de consolidar barrios que pueden ser denominados ilegales o de invasión, el bajo control por autoridades y el ineficiente plan de contingencia para las consecuencias del

Publicado en formato digital: Hugo A. Granados Castillo, Pedro O. Loaiza Parra (Director: Dr. Oscar Luis Pyszczek). EL RUMBO DE LA POBLACIÓN DESPLAZADA EN BOGOTÁ-COLOMBIA. Estudio de caso en la Localidad de Kennedy, 1980-2016. Revista Geográfica Digital. IGUNNE. Facultad de Humanidades. UNNE. Año 14. Nº 28. Julio - Diciembre 2017. ISSN 1668-5180 Resistencia, Chaco.

En: http://hum.unne.edu.ar/revistas/geoweb/default.htm 
conflicto armado fue generando con el pasar de los años un desorden en la distribución y conglomeración de diferentes barrios y localidades.

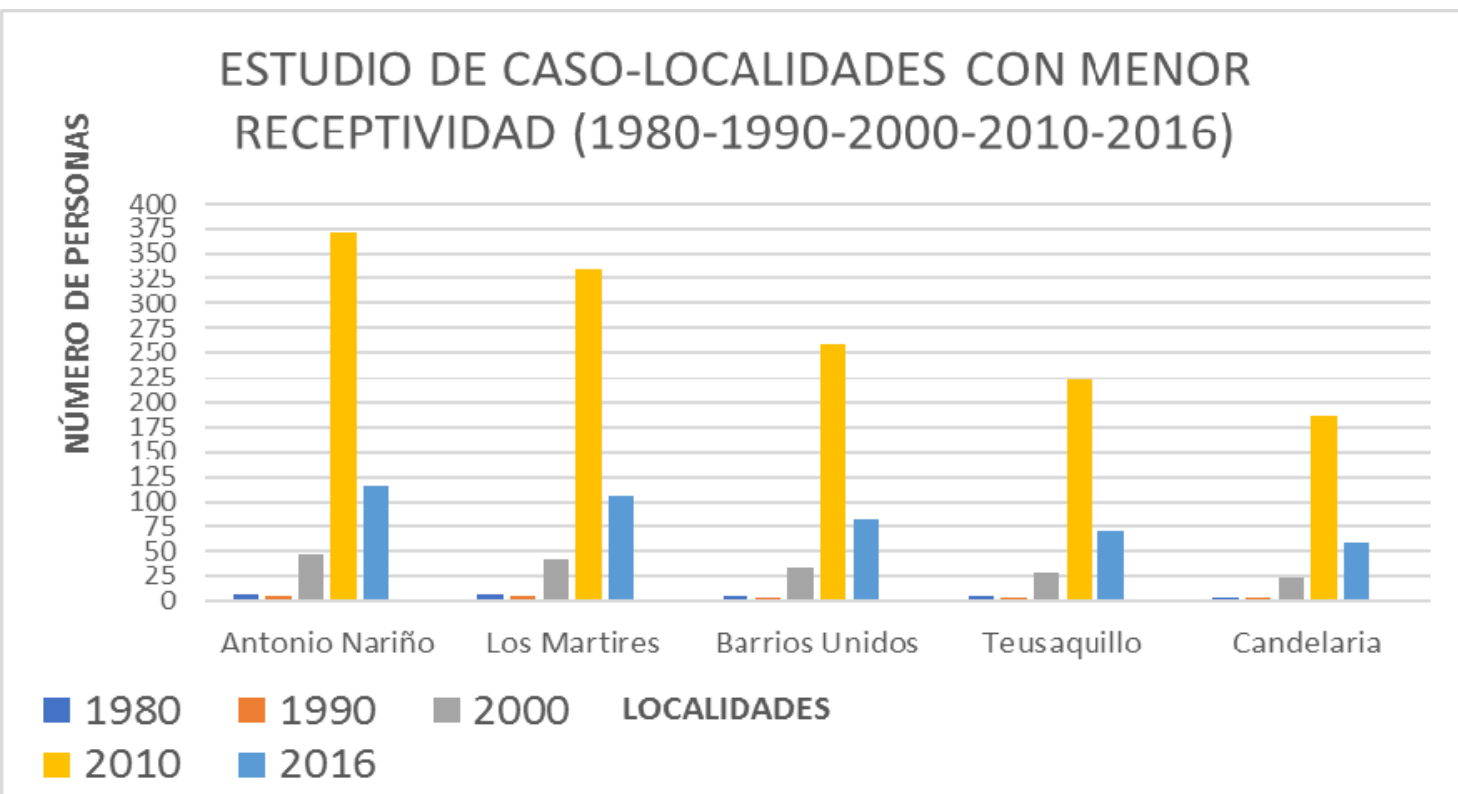

Gráfico 2. Localidades con menor receptividad (Elaboración propia con base en datos de la RUV Obtenido de https://rni.unidadvictimas.gov.co/?q=v-reportes)

El gráfico $\mathrm{N}^{\circ} 2$ se presenta la distribución de la población desplazada recibida en Bogotá desde 1980 al 2016, específicamente para estas localidades (Antonio Nariño, Los Mártires. Barrios unidos, Teusaquillo y La Candelaria), sin embargo, esta comparación local se desarrolló con base a que son localidades que presentan un área menor a $1.419 \mathrm{Ha}$ (perteneciente a Teusaquillo, siendo la más grande entre estas). No obstante, podemos identificar que independientemente de la extensión urbana que tiene cada una de las localidades, no son definidas respectivamente por la cantidad de población desplazada que reciben. Un factor importante a tener en cuenta son las oportunidades que ofrecen estas localidades a nivel individual y la suma de asentamientos legales, como notamos Antonio Nariño fue la localidad más receptora de esta población debido al costo de vivienda y la accesibilidad que tienen las personas para conseguir un empleo informal. Por otro lado, la candelaria presenta menor porcentaje de población desplazada, puesto que, no cuenta con una extensión urbana tan amplia como otras localidades (siendo caracterizada como la más infanta) en efecto no tiene más capacidad receptora para esta población.

Publicado en formato digital: Hugo A. Granados Castillo, Pedro O. Loaiza Parra (Director: Dr. Oscar Luis Pyszczek). EL RUMBO DE LA POBLACIÓN DESPLAZADA EN BOGOTÁ-COLOMBIA. Estudio de caso en la Localidad de Kennedy, 1980-2016. Revista Geográfica Digital. IGUNNE. Facultad de Humanidades. UNNE. Año 14. Nº 28. Julio - Diciembre 2017. ISSN 1668-5180 Resistencia, Chaco.

En: http://hum.unne.edu.ar/revistas/geoweb/default.htm 
Revista Geográfica Digital. IGUNNE. Facultad de Humanidades. UNNE. Año 14. № 28.

Julio - Diciembre 2017. ISSN 1668-5180 Resistencia, Chaco

\section{ESTUDIO DE CASO RECEPTIVIDAD EN LA LOCALIDAD DE KENNEDY Y}

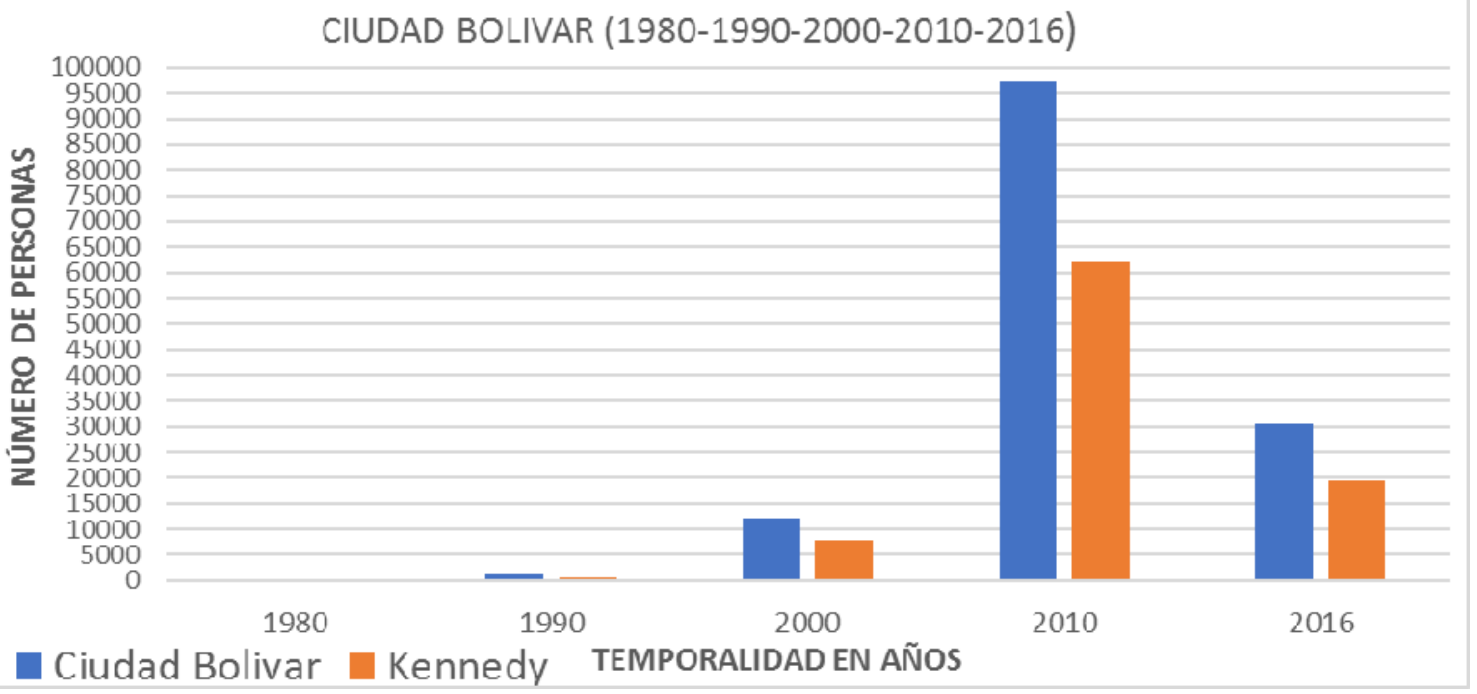

Grafico 3. Localidad con mayor receptividad (Elaboración propia con base en datos de la RUV Obtenido de https://rni.unidadvictimas.gov.co/?q=v-reportes)

La representación estadística de la relación entre la localidad de Kennedy y Ciudad Bolívar junto con la población desplazada aceptada en determinados años, pretende exteriorizar los años donde se presenta mayor receptividad de población desplazada hacia estas localidades, como podemos observar la localidad de Ciudad Bolívar contiene mayor cantidad de desplazados a nivel local en Bogotá, "esta localidad es una de las que presenta mayor índice de pobreza, comprende población entre los estratos 1 y 2" (Beatriz, 2004), siendo de este modo más vulnerable para la recepción de este tipo de población. Sin embargo, para la localidad los índices de pobreza son un poco menores, considerando también esta localidad como la segunda que presenta mayor receptividad de población desplazada, en consideración a esta población y este fenómeno en crecimiento las entidades gubernamentales optaron por la creación de un barrio (Carvajal) el cual fue diseñado con un proyecto de vivienda de interés social para esta población.

Es importante mencionar las condiciones de la localidad respectivamente donde se aloja este tipo de población, también las circunstancias o escenarios a los que son expuestos al llegar allí. Los servicios públicos es una de las condiciones que altera de cierta forma el modo de vida de estas personas puesto que, en la localidad de Ciudad Bolívar la cobertura de servicios públicos no es negativa a nivel general, pero se presentan casos en barrios de invasión o asentamientos ilegales que sufren consecuencias con respecto a la recepción de servicios públicos.

Entre otras variables, encontramos la educación, la cual desempeña un papel importante en esta temática debido a que es significativo conocer con qué nivel de educación llega la población desplazada a la localidad, así mismo, se puede optar por proyectos enfocados para el apoyo de esta población en su ámbito educacional, con el propósito de ofrecer mejores oportunidades a estas personas a nivel laboral y académico, generando como efecto un mejor desarrollo social, económico y cultural, además de brindar una estabilidad para esta población afecta.

Es importante conocer el porcentaje de la población desplazada para estas localidades en relación con la cantidad total de habitantes. Teniendo en cuenta lo anterior, se calculó el

Publicado en formato digital: Hugo A. Granados Castillo, Pedro O. Loaiza Parra (Director: Dr. Oscar Luis Pyszczek). EL RUMBO DE LA POBLACIÓN DESPLAZADA EN BOGOTÁ-COLOMBIA. Estudio de caso en la Localidad de Kennedy, 1980-2016. Revista Geográfica Digital. IGUNNE. Facultad de Humanidades. UNNE. Año 14. Nº 28. Julio - Diciembre 2017. ISSN 1668-5180 Resistencia, Chaco.

En: http://hum.unne.edu.ar/revistas/geoweb/default.htm 
Revista Geográfica Digital. IGUNNE. Facultad de Humanidades. UNNE. Año 14. № 28. Julio - Diciembre 2017. ISSN 1668-5180 Resistencia, Chaco

porcentaje de población desplazada de la localidad de ciudad bolívar sobre el nuero total de habitantes, se tomó la suma total de la población desplazada de todos los años, luego se dividió sobre el numero toral de habitantes y finalmente se sacó el porcentaje, dando como resultado que el $19 \%$ de la población total de la localidad son habitantes desplazados. Respectivamente se realizó el mismo procedimiento para la localidad de Kennedy, siendo el resultado de $7 \%$ de población desplazada sobre el total de habitantes.

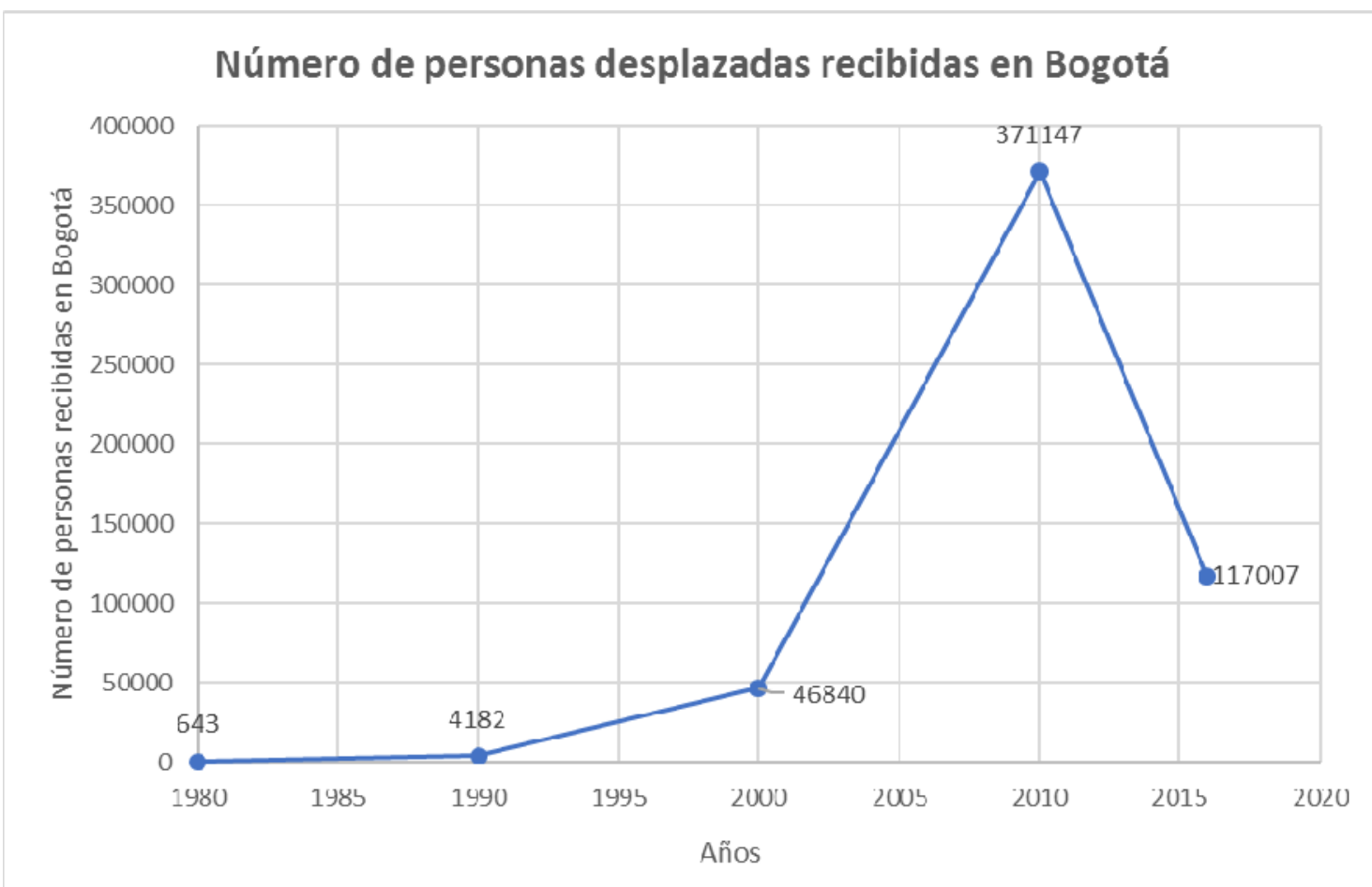

Gráfico 4. Número de personas desplazadas recibidas en Bogotá (elaboración propia con base en datos de la RUV Obtenido de https://rni.unidadvictimas.gov.co/?q=v-reportes)

En el gráfico de línea de tendencia podemos observar el aumento progresivo de receptividad de población desplazada en Bogotá, esto se debe a que "la dinámica del conflicto armado, su reflejo y el posterior establecimiento de la capital como centro de operaciones de los grupos armados no solo para el control de sus enemigos, sino también para consolidar el monopolio sobre las diversas manifestaciones de los negocios ilegales, han determinado un movimiento constante y prácticamente invisible de personas a su interior y hacia sectores aledaños en el área metropolitana" (CODHES, 2014)

En la temporalidad (2001-2010) aumento en gran medida el flujo de población desplazada a Bogotá fue en estos años donde se intensifico el conflicto armado, o al menos donde se empezaron a presenciar casos desconsoladores en las áreas rurales, que no dejo otra opción para esta población que emigrar a las diferentes ciudades y Bogotá al ser la capital fue la ciudad que mayor receptividad presento, este flujo de personas ha intensificado los problemas que presenta una ciudad en desarrollo. Además, esta problemática genero incidencias en la economía de Colombia, según el Ministerio de Ambiente y Desarrollo Sostenible, desde el año 2000 hasta el 2010 se presentó un considerable decrecimiento en la actividad agropecuaria.

Publicado en formato digital: Hugo A. Granados Castillo, Pedro O. Loaiza Parra (Director: Dr. Oscar Luis Pyszczek). EL RUMBO DE LA POBLACIÓN DESPLAZADA EN BOGOTÁ-COLOMBIA. Estudio de caso en la Localidad de Kennedy, 1980-2016. Revista Geográfica Digital. IGUNNE. Facultad de Humanidades. UNNE. Año 14. Nº 28. Julio - Diciembre 2017. ISSN 1668-5180 Resistencia, Chaco.

En: http://hum.unne.edu.ar/revistas/geoweb/default.htm 
La mayoría de los planes, entre ellos el Plan Distrital para la Atención de los Desplazados por la Violencia, parten de la necesidad de realizar un diagnóstico sobre la población desplazada, para luego diseñar e implementar un sistema de información, a fin de identificar las causas de su desplazamiento, su situación socioeconómica, sus necesidades y potencialidades. Esta labor es la base para tomar las medidas de prevención y atención integral, no obstante, la necesidad actual es articular las diferentes instituciones para que así la mirada y las propuestas de solución sean del todo integrales.

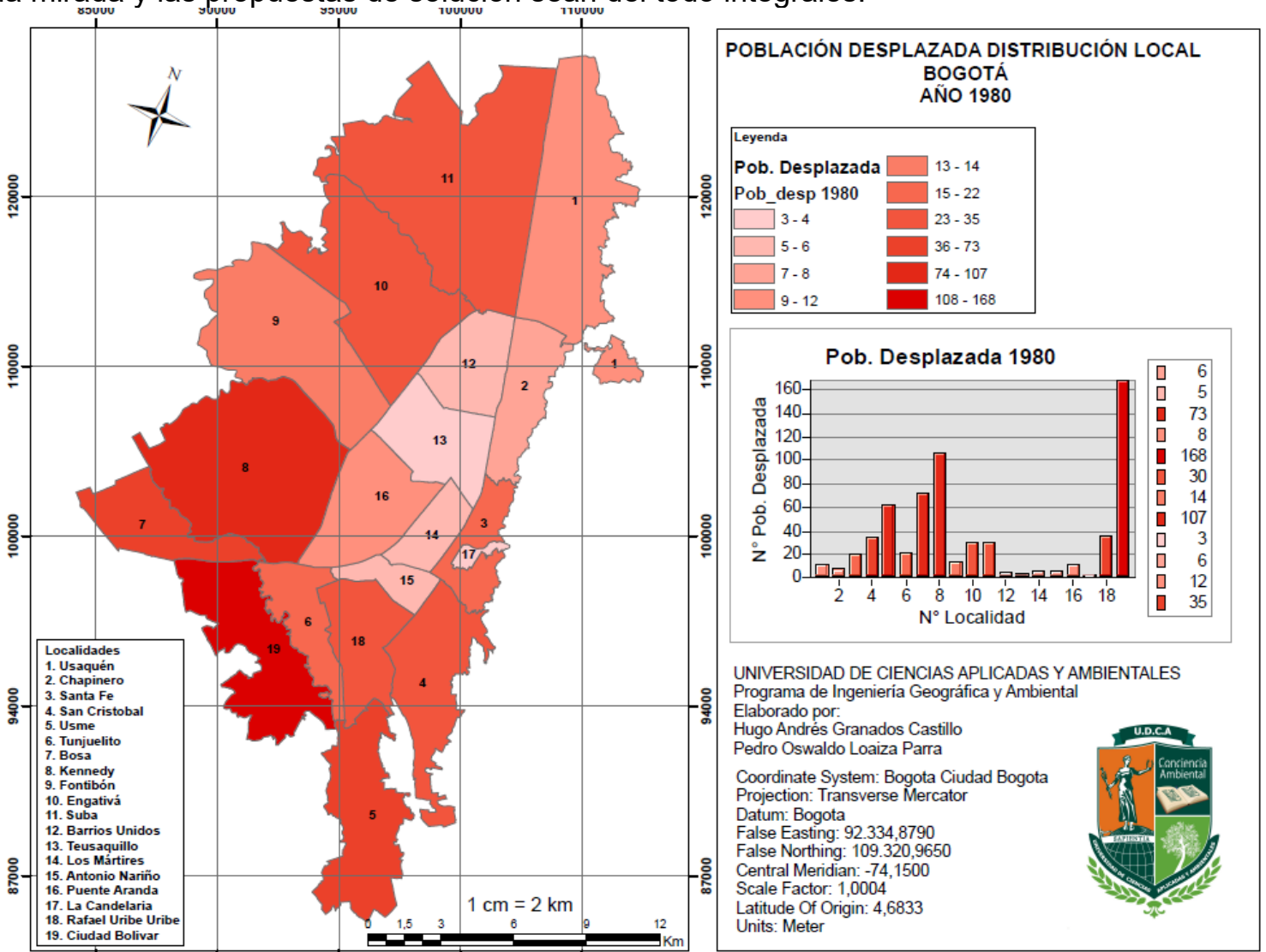

Mapa 2. Distribución local en Bogotá de población desplazada 1971-1980 (Elaboración propia con base en datos URT)

Podemos observar un contraste entre la densidad de población desplazada y el número de personas desplazadas en las diferentes localidades de Bogotá, podemos observar que la mayor receptividad se presenta en las localidades del sur y se prolonga hacía el occidente de la capital. El asentamiento de esta población en las periferias se debe en cierta medida a que en estos sectores no existen grandes limitantes naturales, caso contrario en el oriente de la ciudad con los cerros Orientales. Las localidades con menor receptividad, además de tener áreas pequeñas en comparación con las mencionadas anteriormente tienen una gran densidad poblacional, lo cual limita el ingreso de más población.

Publicado en formato digital: Hugo A. Granados Castillo, Pedro O. Loaiza Parra (Director: Dr. Oscar Luis Pyszczek). EL RUMBO DE LA POBLACIÓN DESPLAZADA EN BOGOTÁ-COLOMBIA. Estudio de caso en la Localidad de Kennedy, 1980-2016. Revista Geográfica Digital. IGUNNE. Facultad de Humanidades. UNNE. Año 14. Nº 28. Julio - Diciembre 2017. ISSN 1668-5180 Resistencia, Chaco.

En: http://hum.unne.edu.ar/revistas/geoweb/default.htm 


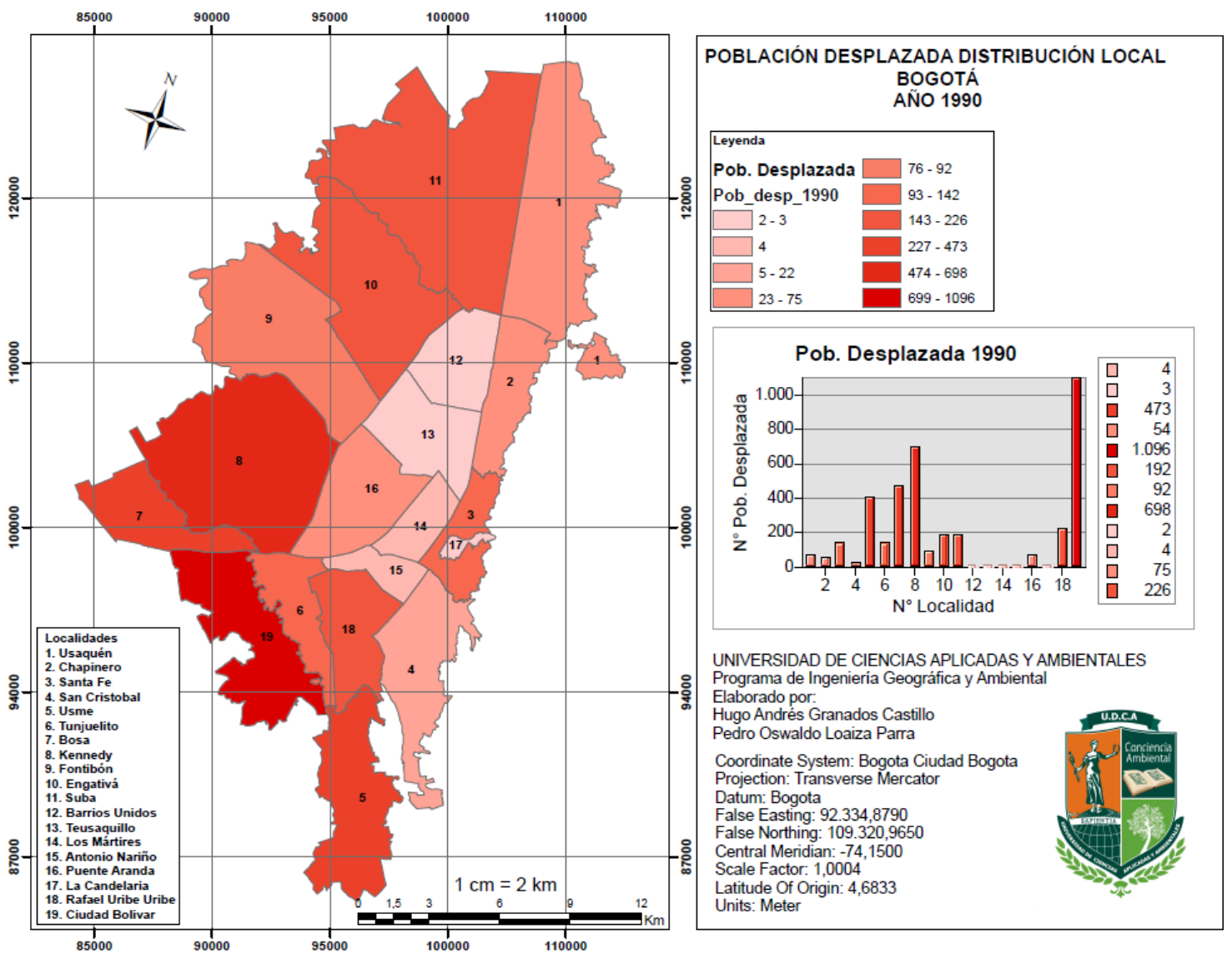

Mapa 3. Distribución local en Bogotá de población desplazada 1981-1990 (Elaboración propia con base en datos URT)

En el mapa número dos (2) observamos que se presenta una dinámica común a la anterior, esto hizo que estas localidades se empezaran a consagrar como barrios receptivos de población desplazada, fue en este momento donde se debieron tomar medidas de contingencia para las consecuencias en las ciudades del conflicto armado, la problemática se fue diversificando aumentando la población en las ciudades principales.

Si bien en esta época se intensifican los grupos al margen de la ley y se consagran con apoyo del narcotráfico, no se presentaba gran retención de tierras, ya que su consolidación se daba en zonas de difícil acceso y poca densidad poblacional. Pyszczek). EL RUMBO DE LA POBLACIÓN DESPLAZADA EN BOGOTÁ-COLOMBIA. Estudio de caso en la Localidad de Kennedy, 1980-2016. Revista Geográfica Digital. IGUNNE. Facultad de Humanidades. UNNE. Año 14. $N^{\circ} 28$. Julio - Diciembre 2017. ISSN 1668-5180 Resistencia, Chaco.

En: http://hum.unne.edu.ar/revistas/geoweb/default.htm 


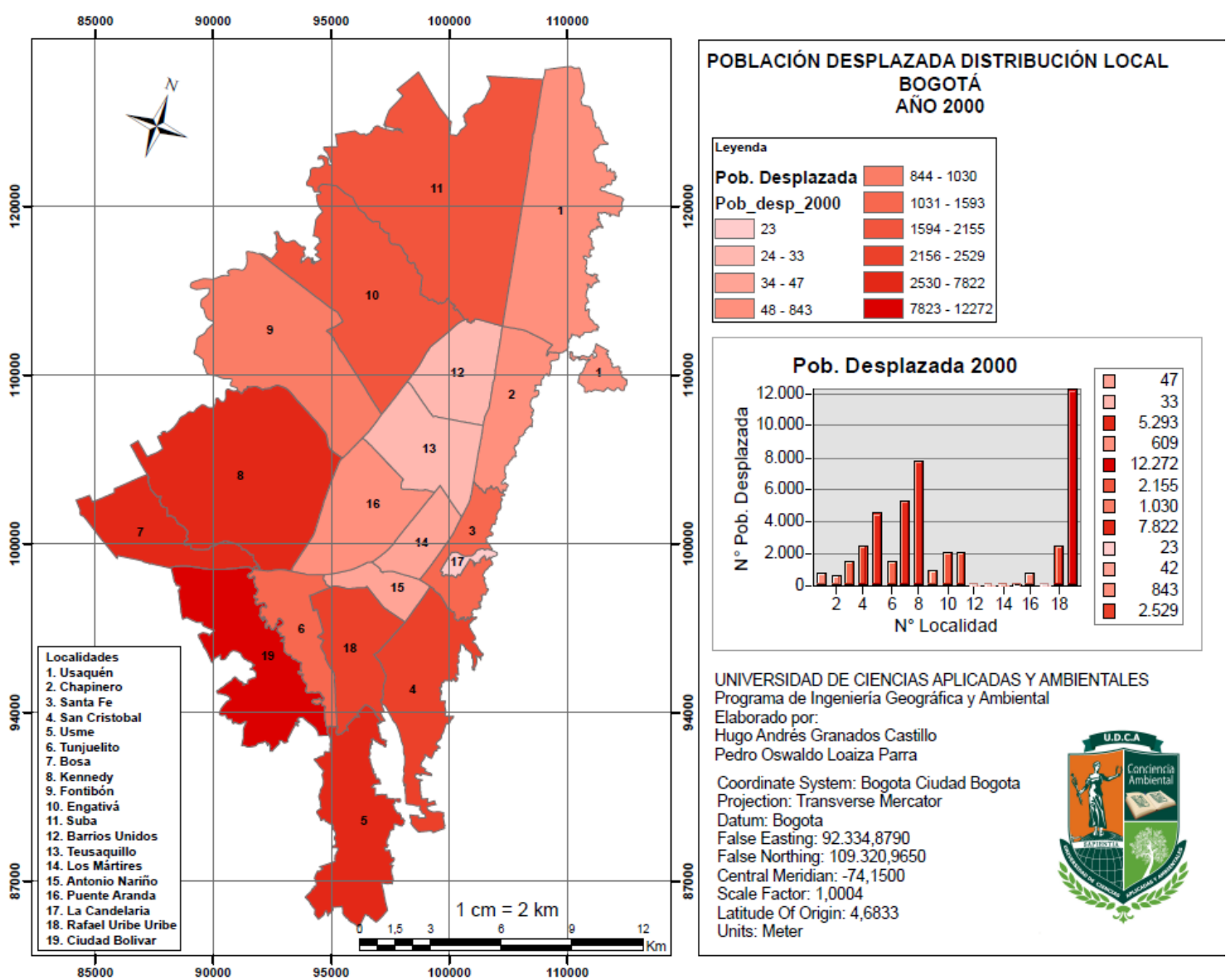

Mapa 4. Distribución local en Bogotá de población desplazada 1991-2000 (Elaboración propia con base en datos URT)

El modelo de desarrollo urbano bajo el cual la ciudad de Bogotá se ha expandido en las últimas décadas ha situado a los sectores poblacionales con menor capacidad adquisitiva en espacios periféricos en los que las condiciones sociales y económicas son notablemente inferiores a las del resto de la ciudad y donde permanecen dificultades relacionadas con la seguridad, el limitado acceso a servicios públicos, problemas ambientales, hacinamiento y distanciamiento de los ejes de desarrollo económico y político. "Es los sectores de la periferia de la capital, donde están los sectores marginales y los actores armados encuentran oportunidades para consolidar su accionar y realizar control social y económico sobre grupos poblacionales con altos niveles de vulnerabilidad." (CODHES, 2014).

En el mapa tres (3) observamos que el crecimiento es continuo y distribuido de la misma manera que en los presentados anteriormente, sin embargo, la problemática ya empieza a generar una gran preocupación para el Estado, por lo cual se empiezan a consolidar diferentes entidades en búsqueda de una solución y atención a la población desplazada en Bogotá y el resto del país.

Publicado en formato digital: Hugo A. Granados Castillo, Pedro O. Loaiza Parra (Director: Dr. Oscar Luis Pyszczek). EL RUMBO DE LA POBLACIÓN DESPLAZADA EN BOGOTÁ-COLOMBIA. Estudio de caso en la Localidad de Kennedy, 1980-2016. Revista Geográfica Digital. IGUNNE. Facultad de Humanidades. UNNE. Año 14. Nº 28. Julio - Diciembre 2017. ISSN 1668-5180 Resistencia, Chaco.

En: http://hum.unne.edu.ar/revistas/geoweb/default.htm 
Revista Geográfica Digital. IGUNNE. Facultad de Humanidades. UNNE. Año 14. № 28.

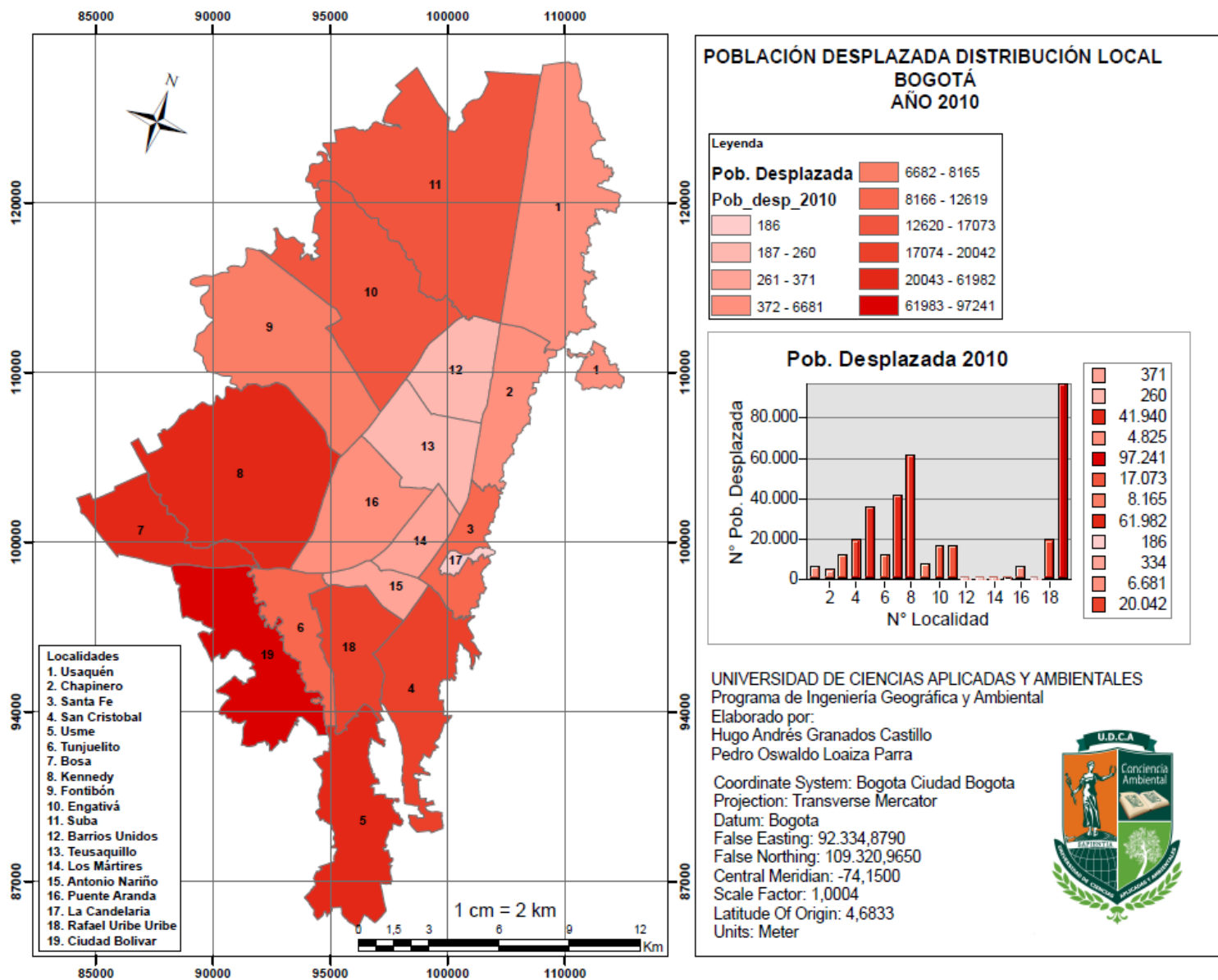

Mapa 5. Distribución local en Bogotá de población desplazada 2001-2010 (Elaboración propia con base en datos URT)

En el mapa cuatro (4) es el que mayor número de población representa, si nos remitimos al gráfico cuatro (4) observamos que es el año en que mayor número de personas han llegado a Bogotá a causa del desplazamiento forzado y en general el conflicto armado generado por parte de los diferentes grupos al margen de la ley. Esto obedece a la gran atracción que ofrece la ciudad como centro político, económico, administrativo y en conclusión de oportunidades, además a la inexistencia de políticas activas que atiendan el desplazamiento en las principales ciudades. Por lo tanto, desde el 2001 hasta la actualidad el desplazamiento hacía Bogotá se configura por el hecho que las zonas rurales o próximas al campo no ofrecen las mismas oportunidades que la capital.

"Vale resaltar, que el período 2001-2003 se caracteriza como el de mayor expulsión de familias, lo que coincide con la ruptura de las negociaciones entre el Gobierno Nacional y las FARC, y con el pulso militar que siguió a la terminación de la zona de despeje del Caguán" (ACNUR, 2004) lo cual responde a los resultados obtenidos en el procesamiento de la información, la dinámica en la distribución de las localidades no varía, por lo cual nos permite caracterizar las áreas del sur y el sur-occidente, como el nuevo albergue o rumbo de la población desplazada en Bogotá. Los planes de acción para el desarrollo social y espacial de esta población se deben realizar desde las alcaldías locales con el apoyo de la alcaldía

Publicado en formato digital: Hugo A. Granados Castillo, Pedro O. Loaiza Parra (Director: Dr. Oscar Luis Pyszczek). EL RUMBO DE LA POBLACIÓN DESPLAZADA EN BOGOTÁ-COLOMBIA. Estudio de caso en la Localidad de Kennedy, 1980-2016. Revista Geográfica Digital. IGUNNE. Facultad de Humanidades. UNNE. Año 14. № 28. Julio - Diciembre 2017. ISSN 1668-5180 Resistencia, Chaco.

En: http://hum.unne.edu.ar/revistas/geoweb/default.htm 
mayor de Bogotá y las diferentes instituciones y unidades constituidas para esta problemática, el punto de partida debe ser la articulación.

La falsa idea de seguridad y oportunidades hizo que su dinámica fuera desfavorable en la mayoría de los casos, según el CODHES es la población desplazada la que mayor vulnerabilidad de los derechos humanos presenta desde los servicios básicos hasta la oportunidad de educación.

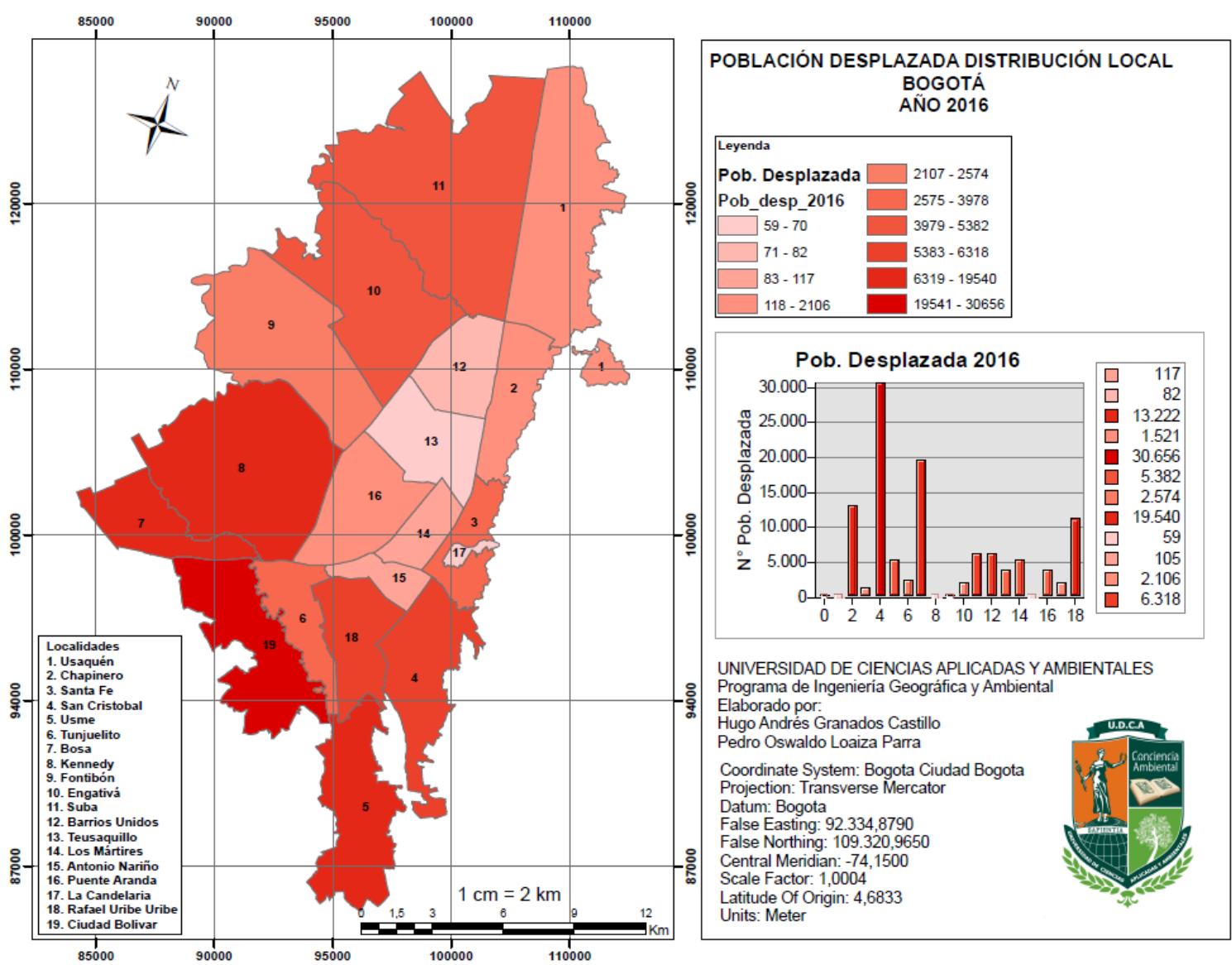

Mapa 6. Distribución local en Bogotá de población desplazada 2001-2010 (Elaboración propia con base en datos URT)

Todos los análisis coinciden en la gravedad del problema, como se puede observar en los diferentes mapas, a partir del 2000 la receptividad en las diferentes localidades de Bogotá empezaron a presentar un aumento significativo, no obstante, en el 2016 se puede observar una disminución, la cual puede ser causa de dos alternativas. En primer lugar, los planes, programas y proyectos inmersos en el plan de desarrollo nacional hace énfasis en buscar la restitución de las tierras para la población que ha sufrido este flagelo, incluso, se han creado diferentes instituciones y unidades públicas con una orientación a esta problemática, por ejemplo; URT (Unidad de Restitución de Tierras), RUV (Registro Único de Victimas), UAID (Unidad de Atención Integral a la Población Desplazada), RSS (Red de Solidaridad Social Unidad Territorial de Bogotá), entre otras. $Y$ de carácter privado como CODHES (Consultoría para los Derechos Humanos y el Desplazamiento), MENCOLDES (Fundación Menonita Colombiana para el Desarrollo), entre otras.

Publicado en formato digital: Hugo A. Granados Castillo, Pedro O. Loaiza Parra (Director: Dr. Oscar Luis Pyszczek). EL RUMBO DE LA POBLACIÓN DESPLAZADA EN BOGOTÁ-COLOMBIA. Estudio de caso en la Localidad de Kennedy, 1980-2016. Revista Geográfica Digital. IGUNNE. Facultad de Humanidades. UNNE. Año 14. Nº 28. Julio - Diciembre 2017. ISSN 1668-5180 Resistencia, Chaco.

En: http://hum.unne.edu.ar/revistas/geoweb/default.htm 
Revista Geográfica Digital. IGUNNE. Facultad de Humanidades. UNNE. Año 14. № 28.

Julio - Diciembre 2017. ISSN 1668-5180 Resistencia, Chaco

En segundo lugar, los datos tratados estadísticamente respondían a una temporalidad de seis (6) años (2011-2016), mientras que en el resto de tratamientos cuantitativos fueron con base en 10 años. No obstante, si consideramos que se trabajó con alrededor de la mitad de los años, podemos percibir que la problemática a futuro aumentará en relación con el análisis temporal de la población desplazada en el período (2001-2010) en caso de seguir con la misma dinámica receptora.

\section{CONCLUSIONES}

Es evidente el alto flujo de población desplazada por conflicto armado en la ciudad de Bogotá, además de la distribución que ostenta esta población a nivel local. No obstante, con respecto a los análisis obtenidos, se logró evidenciar la relación del problema con base en la población desplazada recibida desde los años 1980 hasta 2016 y el crecimiento total de habitantes que presenta cada una de las diferentes localidades. Lo anterior es justificado con un porcentaje que abarca la cantidad de desplazados sobre la totalidad de habitantes, respectivamente para la localidad de Ciudad Bolívar y Kennedy, quienes presentan una mayor receptividad de población víctima del conflicto armado, por lo tanto, dentro de los planes de Desarrollo y Acción de estas localidades, se deben implementar proyectos y programas que aseguren el respeto por la dignidad y derechos humanos en general.

Sin embargo, con los datos provistos por la Unidad de Atención a Víctimas sobre la población desplazada de las diferentes localidades de Bogotá, se identificó después del procesamiento de datos, la generación de cartografía y estadísticas, que la problemática del desplazamiento generado en consecuencia del conflicto armado en la capital es una cuestión que comprende no solo la capital sino el territorio a nivel nacional, haciendo referencia a los departamentos y municipios expulsores. De acuerdo con lo anterior se requiere un control y seguimiento por parte de las entidades públicas y privadas, con el fin de adoptar policías, planes, programas y proyectos donde se relacione la capital de Bogotá (siendo reconocida evidentemente como la ciudad más receptora de población desplazada), los departamentos y municipios expulsores. Obteniendo como designio mitigar la concentración y el movimiento de población desplazada hacia la capital.

El tipo de desplazamiento individual ocasiona una mirada desarticulada de la problemática, por lo tanto, resulta fundamental para la solución de esta articular desde las alcaldías locales hasta las diferentes entidades delegadas por el estado en el marco de las consecuencias del conflicto armado. Esto permitirá una mirada desde lo particular hasta lo general, ya que, como observamos en la cartografía y los gráficos de barras, el problema se agudiza en el sur occidente de la ciudad, pero sus afectaciones resultan involucrando la capital en general. Por otro lado, las entidades del estado relacionadas con el tema de población desplazada presentan diferentes adversidades para poner sobre prioridad este problema de desplazamiento, así como hay otras que ni siquiera lo determinan, en otras pablaras es considerado un problema de "otros" ya que no son afectados, también, desisten a un compromiso con la cantidad de personas afectadas por este problema que llegan a la capital. Con la presente dificultad, la defensoría del pueblo habla de las políticas públicas que deben dirigirse al restablecimiento de la dignidad de la población afectada, así como la restauración de sus derechos afectados relacionados directamente con la condición de vulnerabilidad.

El desplazamiento forzado en Colombia exige una coordinación y concurrencia por parte de la Nación, una política activa en la capital que implique un esfuerzo de gran magnitud, debido a que la problemática seguirá generando repercusiones y su dimensión aumentará conforme pase el tiempo, de continuar la misma "ruta de desplazamiento", se aconseja un control de la población víctima de este flagelo y así mismo una previa planeación del territorio que actuará como receptor.

Publicado en formato digital: Hugo A. Granados Castillo, Pedro O. Loaiza Parra (Director: Dr. Oscar Luis Pyszczek). EL RUMBO DE LA POBLACIÓN DESPLAZADA EN BOGOTÁ-COLOMBIA. Estudio de caso en la Localidad de Kennedy, 1980-2016. Revista Geográfica Digital. IGUNNE. Facultad de Humanidades. UNNE. Año 14. N² 28. Julio - Diciembre 2017. ISSN 1668-5180 Resistencia, Chaco.

En: http://hum.unne.edu.ar/revistas/geoweb/default.htm 
Revista Geográfica Digital. IGUNNE. Facultad de Humanidades. UNNE. Año 14. № 28.

Julio - Diciembre 2017. ISSN 1668-5180 Resistencia, Chaco

\section{REFERENCIAS}

- Agencia de los Estados Unidos para el Desarrollo Internacional (USAID). (2015). Memoria Encuentro Regional sobre Desplazamiento Forzado por Violencia. Tegucigalpa, Honduras: CIPRODEH.

- Alto Comisionado de las Naciones Unidas para los Refugiados. (2012). Acnur. Obtenido de Desplazamiento Forzado en Colombia: http://www.acnur.org/fileadmin/scripts/doc.php?file=fileadmin/news_imported_files/C Ol_2821

- GÓMEZ, J. T. (2011). DESPLAZAMIENTO FORZOSO EN COLOMBIA: EXPULSIÓN Y MOVILIDAD, DOS DINÁMICAS QUE INTERACTÚAN. Maestria en Ciencias Económicas. Bogotá: Universidad Nacional de Colombia.

- M, V. D. (2009). La Gente de Nadie: Desplazamiento Forzado. Obtenido de http://repository.urosario.edu.co/bitstream/handle/10336/1256/1019005462.pdf;jsessi onid=D4401A5E47C02E3D21FD38764F8A455B? sequence $=1$

- Ministerio de Defensa. (s.f.). Mindefensa. Obtenido de Desplazamiento Forzado: https://www.mindefensa.gov.co/irj/go/km/docs/Mindefensa/Documentos/descargas/D ocumentos_Descargables/espanol/Desplazamiento\%20Forzado.pdf

- Naciones Unidas Derechos Humanos. (2013). Derechos Humanos. Nueva York: Oficial del Alto Comisionado.

- ONU. (2016). Colombia es el país con mayor desplazamiento forzados en el mundo: ONU . California.

- Pérez, A. S. (2004). Acceso de la población desplazada por conflicto armado a los servicios de salud en las empresas sociales del estado de primer nivel de la ciudad de Bogotá-Colombia. Obtenido de http://ddd.uab.cat/pub/tesis/2004/tdx-0620105134834/asmp1de1.pdf

- Registro único de víctimas. (28 de Diciembre de 2016). UnidadVictimas. Obtenido de https://rni.unidadvictimas.gov.co/?q=v-reportes

- ACNUR. (2004). Una realidad de clama atención. Alcaldía Mayor de Bogotá.

- Beatriz, L. T. (2004). Bogotá: una ciudad receptora de migrantes y desplazados con graves carencias en materia de recursos y de institucionalidad para garantizarles sus derechos. SciElo, 3.

- CODHES. (2014). Desplazamiento Intraurbano y Soluciones Duraderas. In M. Romero, Desplazamiento Intraurbano y Soluciones Duraderas Volumen II (p. 17). Bogotá: CODHES.

- Infraestructura de datos espaciales para el distrito capital. (2017). IDECA. Obtenido de https://www.ideca.gov.co/es/servicios/mapa-de-referencia/tabla-mapa-referencia

Publicado en formato digital: Hugo A. Granados Castillo, Pedro O. Loaiza Parra (Director: Dr. Oscar Luis Pyszczek). EL RUMBO DE LA POBLACIÓN DESPLAZADA EN BOGOTÁ-COLOMBIA. Estudio de caso en la Localidad de Kennedy, 1980-2016. Revista Geográfica Digital. IGUNNE. Facultad de Humanidades. UNNE. Año 14. N 28. Julio - Diciembre 2017. ISSN 1668-5180 Resistencia, Chaco.

En: http://hum.unne.edu.ar/revistas/geoweb/default.htm 